\title{
Integrated analysis using methylation and gene expression microarrays reveals PDE4C as a prognostic biomarker in human glioma
}

\author{
ZHAOSHI BAO $^{1,4,5}$, YING FENG ${ }^{2}$, HONGJUN WANG ${ }^{3}$, CHUANBAO ZHANG $^{1,4,5}$, \\ LIHUA SUN $^{6}$, ZHUOHONG YAN ${ }^{2}$, QINGYANG LIU ${ }^{2}$, TIANZHU GUO ${ }^{2}$, MINGYANG LI ${ }^{1,4,5}$, \\ XUE YANG $^{2}$, CHUANLU JIANG ${ }^{3}$, QUANGENG ZHANG ${ }^{2}$ and TAO JIANG ${ }^{1,4,5}$ \\ ${ }^{1}$ Department of Neurosurgery, Beijing Tiantan Hospital, Capital Medical University, Beijing 100050; \\ ${ }^{2}$ Department of Immunology, Institute of Basic Medical Sciences, Capital Medical University, Beijing 100069; \\ ${ }^{3}$ Department of Neurosurgery, The Second Affiliated Hospital of Harbin Medical University, Harbin 150086; \\ ${ }^{4}$ Beijing Neurosurgical Institute, Beijing 100050; ${ }^{5}$ Beijing Insitute for Brain Disorders Brain Tumor Center, Beijing 100050; \\ ${ }^{6}$ Department of Neurosurgery, The Affiliated Wuxi People's Hospital of \\ Nanjing Medical University, Wuxi, Jiangsu 214023, P.R. China
}

Received January 17, 2014; Accepted March 22, 2014

DOI: $10.3892 / o r .2014 .3176$

\begin{abstract}
Hypermethylation of tumor suppressor promoters is generally accepted to indicate poor prognosis in glioma; however, the DNA methylation patterns associated with different glioma prognoses remain to be elucidated. In the present study, promoter methylation and gene expression microarrays were used to screen candidate genes between different grades of glioma. Survival analysis was performed using the Kaplan-Meier (KM) method. Promoter methylation and protein expression of phosphodiesterase 4C (PDE4C) was examined in different grade gliomas and the correlation between PDE4C and wild-type (WT) p53 was evaluated in glioma cell lines. In addition, gene ontology and gene set variation analysis were used to examine PDE4C function. We found PDE4C exhibited promoter hypermethylation in high-grade glioma samples and hypomethylation in low-grade glioma, with PDE4C expression levels showing the reverse. This indicated PDE4C may be a candidate glioma biomarker. Through studies of PDE4C methylation and expression status in an independent cohort of 124 patient samples (56 low-grade and 63 high-grade glioma and 5 normal brain), we identi-
\end{abstract}

Correspondence to: Professor Quangeng Zhang, Department of Immunology, Institute of Basic Medical Sciences, Capital Medical University, 10 Xitoutiao, Youanmen, Fengtai, Beijing 100069, P.R. China

E-mail: quangengzhang@163.com

Professor Tao Jiang, Department of Neurosurgery, Beijing Tiantan Hospital, Capital Medical University, 6 Tiantan Xili, Dongcheng, Beijing 100050, P.R. China

E-mail: taojiang1964@163.com

Key words: hypermethylation, PDE4C, glioma, prognosis, apoptosis fied PDE4C as having significant promoter methylation and lower expression in high-grade glioma. Hypermethylation and reduced PDE4C protein expression were associated with grade progression and overall survival. In glioma cell lines, PDE4C was upregulated by demethylation treatment with 5-Aza-2'deoxycytidine and WT p53 expression was downregulated after PDE4C siRNA suppression. Finally, we found PDE4C promoted apoptosis and inhibited migration in a U87 cell line. On the basis of these observations and the results from subset analysis, it is reasonable to conclude that PDE4C may function as a tumor suppressor by promoting apoptosis through the WT p53 pathway and inhibiting cell migration. The data show that PDE4C is downregulated through promoter hypermethylation in glioma.

\section{Introduction}

Glioma is the most common primary brain tumor in adults (1). For patients with glioblastoma, the most aggressive glioma, the survival time after diagnosis averages 10-14 months, despite improvements in available treatments, such as surgery and radiotherapy with temozolomide (TMZ) $(1,2)$.

Glioma progression is caused by an accumulation of genetic and epigenetic alterations; therefore, individualized diagnosis and treatments that focus on specific genes are essential (3). Previous studies have shown that the expression of genes related to glioma progression include COL4A2, FOXM1, MGP, TOP2A, CENPF, IGFBP4, VEGFA, ADD3, CAMK2G, KI-67, YKL-40 and SMAD4, all of which are associated with the World Health Organization (WHO) glioma grade (4-6). Moreover, gene methylation is believed to play a crucial role in tumor progression, partially by repressing tumor suppressor genes, inhibiting their accumulation and inducing their mutation $(7,8)$. Conversely, prognostic markers with potential clinical significance have been identified through genome- 
wide DNA methylation microarrays and survival-associated gene expression profiling studies (2,9-11).

In the present study, using microarray databases, we identified that the human cyclic adenosine monophosphate (cAMP)-specific phosphodiesterase 4C (PDE4C) promoter methylation and expression levels were associated with glioma grade progression. We screened out PDE4C from other genes due to its better survival index.

PDE4C, localized to 19p13.1, belongs to the PDE family. Cyclic nucleotide PDEs hydrolyze the second messengers cAMP and cGMP, which are viewed as critical regulators of many important physiological processes, such as intracellular cAMP levels, cAMP signaling and signal compartmentalization (12). PDEs are grouped into seven enzyme families according to their substrate specificity and sensitivity to pharmacological inhibitors $(13,14)$, with the type 4 PDEs specific for cAMP. The distribution and abundance of PDE4C mRNAs appear to be more restricted than the other three isoforms (PDE4A, B, D) and are largely limited to cell lines of neuronal origin. Pérez-Torres et al (15) compared the localization of each isozyme in the human brain with that in the rat and monkey brain. They found that the four PDE4 isoforms display a differential expression pattern at both regional and cellular level in the three species. PDE4A, PDE4B and PDE4D are widely distributed in the human brain, with the two latter appearing more abundantly. In contrast, PDE4C in the human brain presents a more restricted distribution, limited to cortex, some thalamic nuclei and cerebellum. This is at variance with the distribution of PDE4C in rat and monkey brain. To date, there are no reports on PDE4C correlation with other tumors, and its function remains unclear, except it hydrolyzes human cAMP.

Our data showed that PDE4C which specifically hydrolyzes human cAMP, may be a tumor suppressor gene that is involved in glioma development. Furthermore, previous studies have shown that activation of cAMP signaling inhibits DNA damage-induced apoptosis in BCP-ALL cells through abrogation of p53 accumulation and promotion of p53 and HDM2 interaction $(8,16)$. However, PDE4C remains a putative tumor suppressor, as it has not been identified in gliomas. In the present study, we found that PDE4C CpG island hypermethylation and low PDE4C expression in human glioblastomas were associated with a poor prognosis. In addition, PDE4C underwent $\mathrm{CpG}$ island promoter-associated silencing in glioma, which upon reintroduction into glioblastoma cell lines, promoted apoptosis and inhibited migration. Thus, our findings indicate that PDE4C is a candidate tumor suppressor gene and may serve as a prognostic biomarker with potential clinical significance in glioma treatment.

\section{Materials and methods}

Patients and samples. Patients were from the Chinese Glioma Genome Atlas (CGGA) database (http://www.cgga.org.cn). All patients in the present study underwent surgical resection between January 2006 and December 2010 and subsequently received TMZ and radiotherapy. Clinical data, including patient age at diagnosis, gender, extent of resection and preoperative Karnofsky Performance Status (KPS) score were obtained from medical records. Overall survival (OS) time, defined as the period from operation to mortality, was collected mainly when patients visited the clinics or by phone interview with patients and/or their relatives. Patients who died of non-primary diseases were excluded (17). Tumor tissue samples were obtained by surgical resection before treatment with radiation and chemotherapy. Resected specimens were snap frozen in liquid nitrogen and stored at $-80^{\circ} \mathrm{C}$ until nucleic acid extraction. This study was approved by the Ethics Committee of the Capital Medical University, and written informed consent was obtained from all patients. Two neuropathologists classified the samples according to $2007 \mathrm{WHO}$ classification.

DNA extraction and genome-wide DNA methylation profiling. Hematoxylin and eosin-stained frozen sections were prepared for the assessment of the percentage of tumor cells before DNA extraction. Only samples with $>80 \%$ tumor cells were selected. Genomic DNA was isolated from frozen tumor tissues using the QIAamp DNA Mini kit (Qiagen, Hilden, Germany) according to the manufacturer's protocol. DNA concentration and quality were measured using the NanoDrop ND-1000 spectrophotometer (NanoDrop Technologies, Houston, TX, USA).

A series of 119 glioma samples (63 low-grade gliomas, 33 anaplastic gliomas and 23 glioblastomas) were measured by methylation microarray. We used the Illumina Infinium Human Methylation 27K BeadChip (Illumina Inc., San Diego, CA, USA) as described (18). The BeadChip contains 27,578 highly informative $\mathrm{CpG}$ sites covering $>14,475$ human RefSeq genes. This allows researchers to interrogate all sites per sample at single nucleotide resolution. Bisulfite modification of DNA, chip processing and data analysis were performed following the manufacturer's instructions. Array results were analyzed with the BeadStudio software (Illumina Inc.). The methylation data were uploaded to the CGGA database (http://www.cgga.org.cn).

RNA extraction and gene expression profiling. Frozen sections were stained with hematoxylin and eosin following standard protocols and examined using light microscopy. Samples were reviewed prior to RNA extraction and confirmed to contain $\geq 80 \%$ tumor cells. Total RNA from frozen tumor tissue was extracted using the mirVana miRNA isolation kit (Ambion, Austin, TX, USA) according to the manufacturer's protocol. RNA quantity, quality and integrity were verified using the NanoDrop ND-1000 spectrophotometer and the Agilent Bioanalyzer 2100 (Agilent, Santa Clara, CA, USA).

Microarray analysis was performed on the same cohort of 119 glioma samples measured by methylation microarray using Agilent Gene Expression Oligo Microarrays containing $>41,000$ probe sets for $\sim 27,958$ human genes. Processing was performed according to the Agilent One-Color MicroarrayBased Gene Expression Analysis Protocol. Average values of replicate spots for each gene were background subtracted, normalized, $\log _{2}$-transformed, and subjected to further analysis. The expression data were also uploaded to the CGGA database (http://www.cgga.org.cn).

Pyrosequencing analysis of PDE4C. Pyrosequencing was performed by Gene Tech (Shanghai, China) using the PyroMark Q96 ID System (Qiagen), according to the manufacturer's protocol. Bisulfite modification of the DNA was performed using the EpiTect kit (Qiagen). The primers 5'-GGG GTT TTA TTA 
TGT TGG TTA GGA T-3' and 5'-biotin-TCA AAA ACC CTC TCT AAT CAT TAA-3' were used for polymerase chain reaction (PCR) amplification and the primer 5'-TTA TTA TGT TGG TTA GGA TG-3' for pyrosequencing. The PDE4C promoter was considered hypermethylated when methylation was $>80 \%$ and hypomethylated when methylation was $<20 \%$ ( $\mathrm{P}<0.001)$.

Immunohistochemistry. Immunohistochemistry was performed as previously described (19). Briefly, surgical biopsies were fixed in formalin, processed and paraffin embedded. Five micron-thick sections were prepared, and immunohistochemical staining with streptavidin-biotin immunoperoxidase assay was performed using a rabbit polyclonal antibody to PDE4C (OriGene, Rockville, MD, USA). Negative controls were obtained by substituting primary antibodies with nonimmune serum. The degree of immunostaining was viewed and scored separately by two independent investigators. Scores were determined by the proportion of positively stained tumor cells (2). Sections with $<30 \%$ labeled cells indicated low PDE4C expression, whereas sections with labeling of $\geq 30 \%$ indicated high PDE4C expression.

Gene ontology analysis of PDE4C-associated genes. After Pearson correlation analysis, gene ontology (GO) analysis of positively correlated genes $(r>0.4, \mathrm{P}<0.05)$ was analyzed by DAVID (http://david.abcc.ncifcrf.gov/home.jsp).

Gene set variation analysis with PDE4C expression. With the gene lists obtained from the GO terms (GO:0043065 and GO:0030335), gene set variation analysis (GSVA) was performed in $\mathrm{R}$.

Cell lines and drug treatments. The glioblastoma cell lines U87MG, U251 and H4 were purchased from the Chinese Academy of Sciences Cell Bank (Shanghai, China), and were maintained in Dulbecco's modified Eagle's medium (DMEM) supplemented with L-glutamine and $10 \%$ fetal bovine serum (FBS) (both from HyClone, Waltham, MA, USA). Cells were treated with $5 \mu$ M 5-Aza-2'-deoxycytidine (5-Aza-dC; Sigma, St. Louis, MO, USA) for 4 days (a fresh drug was added every $24 \mathrm{~h}$ ), where indicated.

Transfection of small interfering RNA and plasmids. The small interfering RNA (siRNA) duplexes for targeted silencing of PDE4C were prepared using the Trilencer-27 siRNA kit (OriGene). Briefly, $1 \times 10^{5}$ U87 cells were plated and grown in 6-well plates $24 \mathrm{~h}$ before transfection. When cells were at $70 \%$ confluence, $800 \mathrm{ml}$ Opti-MEM (Life Technologies, San Diego, CA, USA) were added per well, and cells were serum starved for $40 \mathrm{~min}$. siRNA1, the most efficient of the three siRNAs screened, was used to knock down PDE4C (sequence, 5'-ACAAUCAAGCUCUUAGUUAUAGGTG-3'). A blast search of the human genome database was carried out to ensure that the sequence would not target other transcripts. For each well, $0.1 \mathrm{nM}$ siRNA1 was mixed with $5 \mu \mathrm{l}$ of Lipofectamine 2000 (Invitrogen, Carlsbad, CA, USA) in $250 \mu \mathrm{l}$ of Opti-MEM. The mixture was incubated for $30 \mathrm{~min}$ at room temperature and then added to cells. Serum was added $4 \mathrm{~h}$ later to a final concentration of $10 \%$. Total protein was collected for western blot analysis after $48 \mathrm{~h}$.
PDE4C2, as one of the transcripts of human PDE4C, was chosen to carry out our experiments. Previous studies have shown that PDE4C2 expressed by COSI cells have typical bioactivity and bind to rolipram (20). The PDE4C2 expression plasmid was constructed by cloning the full-length PDE4C2 open reading frame into the eukaryotic expression vector pCMV6-Entry, with $S g f \mathrm{I}$ and $M l u \mathrm{I}$ restriction enzyme sites (OriGene). Plasmid construct sequences were confirmed by DNA sequencing. Cells were cultured in 6-well plates for $24 \mathrm{~h}$ and transfected with pCMV6-Entry-PDE4C or empty vector pCMV6-Entry, using Lipofectamine 2000. The primer pairs were designed as previously described, and PCR and DNA sequencing confirmed all constructs.

Reverse transcription-polymerase chain reaction. PDE4C DNA fragments $(-713$ to $-1,207)$ were amplified by PCR using U87, U251 and H4 genomic DNA as the template. RNA was isolated using TRIzol (Invitrogen). One microgram of RNA was reverse transcribed using Moloney murine leukemia virus (M-MLV) (Takara, Shiga, Japan) and amplified using specific primers. The primer pairs were designed as follows: forward, 5'-GGC CTC CAA CAA GTT CAA GCG G-3' and reverse, 5'-CAC GTC GTG GAT GGC GCT TGC-3'. PCR was carried out for $21 \mathrm{cycles}\left(98^{\circ} \mathrm{C}\right.$ for $10 \mathrm{sec}, 68^{\circ} \mathrm{C}$ for $\left.60 \mathrm{sec}\right)$ in a final volume of $25 \mu \mathrm{l}$ containing 5X PrimeSTAR GXL Buffer ( $\mathrm{Mg}^{2+}$ Plus), dNTP mixture (2.5 mM each), and $0.25 \mu \mathrm{l}$ PrimeSTAR GXL DNA Polymerase $(1.25 \mathrm{U} / \mu \mathrm{l})$. Random reverse transcription-PCR (RT-PCR) primers contained a nonadeoxyribonucleotide mixture; pd(N)9 (Takara). Glyceraldehyde-3-phosphate dehydrogenase was used as an internal control to ensure cDNA quality and loading accuracy. Amplification products were resolved by $1.5 \%$ agarose gel electrophoresis and visualized by ethidium bromide staining.

Western blotting. Cells were directly lysed in NP-40 buffer on ice-containing protease inhibitors. Protein lysates were resolved on $10 \%$ SDS polyacrylamide gel, and electrotransferred to PVDF membranes (Millipore, Bedford, MA, USA), and blocked with 5\% nonfat dry milk in Tris-buffered saline, $\mathrm{pH}$ 7.5. Membranes were immunoblotted overnight at $4^{\circ} \mathrm{C}$ with an anti-PDE4C polyclonal antibody (OriGene), or a p53 antibody that selectively recognizes WT p53 protein (Oncogene Research Products, Uniondale, NY, USA), monoclonal anti- $\beta$ tubulin (Sigma) was used as a control. All primary antibodies were followed by their respective HRP-conjugated secondary antibodies.

Flow cytometric analysis of apoptosis. Apoptosis was examined using a fluorescein isothiocyanate (FITC) Annexin V apoptosis detection kit (Becton-Dickinson, San Jose, CA, USA) according to the manufacturer's instructions. Briefly, $1 \times 10^{4}$ U87 cells were harvested and washed with cold PBS. The cells were resuspended in $250 \mu$ of $1 \mathrm{X}$ binding buffer, from which $195 \mu \mathrm{l}$ were transferred to a $1.5-\mathrm{ml}$ culture tube and $5 \mu \mathrm{l}$ of Annexin V-FITC and $10 \mu \mathrm{l}$ propidium iodide were added. Cells were vortexed and incubated for $10 \mathrm{~min}$ in the dark. Three hundred microliters of $1 \mathrm{X}$ binding buffer was then added to each tube.

Flow cytometric analysis was performed immediately after staining. Data acquisition and analysis were performed 


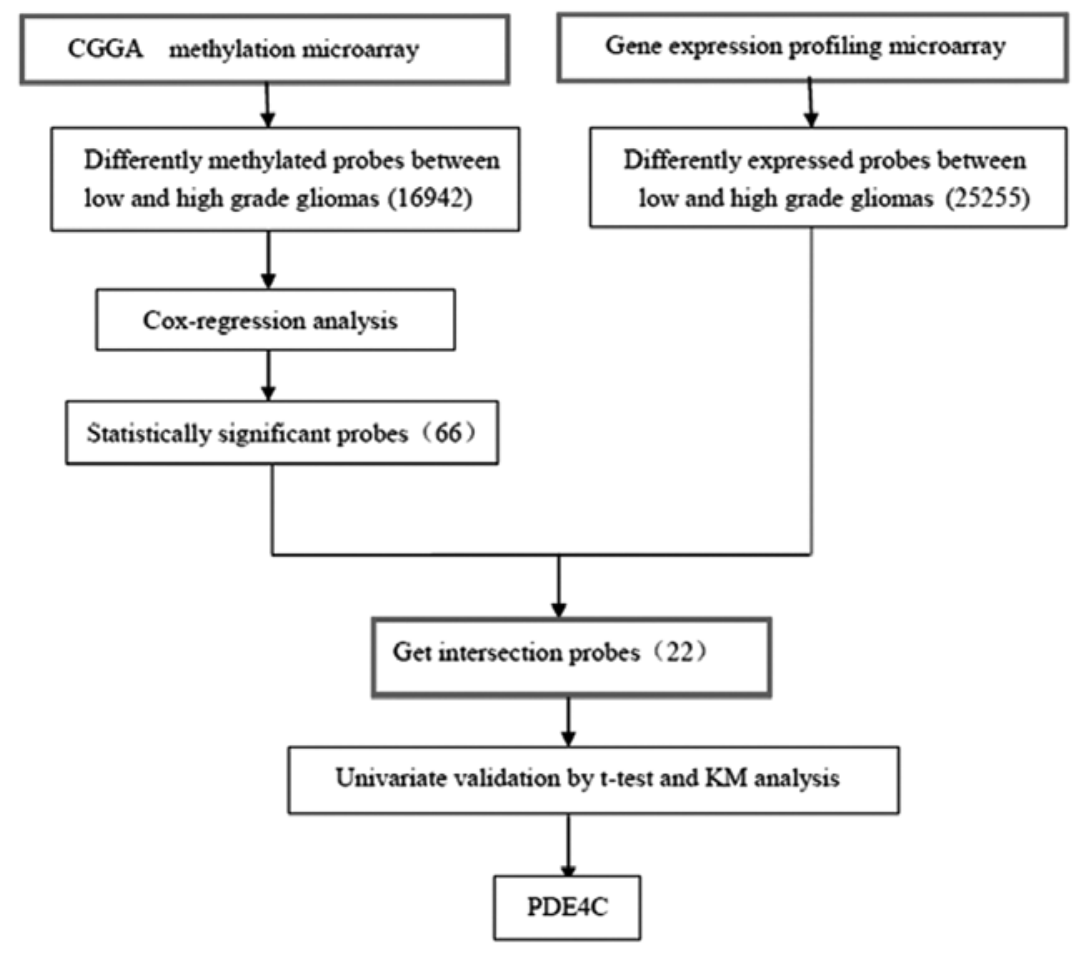

Figure 1. Work flow. CGGA methylation microarray, differential methylation between high- and low-grade gliomas, Cox regression analysis, univariate validation by t-test and log-rank test and $\mathrm{CpG}$ island searches were used to identify genes of interest, including PDE4C. CGGA, Chinese Glioma Genome Atlas; PDE4C, phosphodiesterase 4C.

by fluorescence-activated cell scanner flow cytometer (Becton-Dickinson). Cells in the early stages of apoptosis were Annexin V-positive and PI-negative, whereas cells in late-stage apoptosis were positive for both Annexin V and PI.

Cell migration assay. The cell migration assay was carried out in 24-well cell culture chambers using Transwell inserts (Corning Life Sciences, Corning, NY, USA) with an 8- $\mu$ pore membrane precoated with Matrigel (BD Biosciences, San Jose, CA, USA). U87 cells were plated at a density of $5 \times 10^{3}$ per upper well in $200 \mu \mathrm{l}$ culture medium (DMEM, no FBS), for the empty plasmid group and the PDE4C group. The lower chamber was filled with $500 \mu 1$ medium (DMEM, 12\% FBS). The cells were allowed to invade for $24 \mathrm{~h}$, after which the non-migrating cells with Matrigel matrix were removed from the upper surface of the membrane by scrubbing with a cottontipped swab. Cells on the lower surface of the filter were fixed for $30 \mathrm{~min}$ in methanol and glacial acetic acid mixture (3:1), air-dried briefly, and stained with crystal violet. The mean number of invaded cells was counted from five preselected microscopic fields at x200 magnification. All experiments were performed in triplicate.

Statistical analysis. Significance analysis of microarrays (SAM) was used for genes differentially methylated and expressed between high- and low-grade glioma. Cox regression analysis was performed using Matlab (MathWorks, Natick, MA, USA). Kaplan-Meier survival curves were obtained, and differences in OS were tested using the log-rank test (GraphPad Prism 5). Differences of tumor cell apoptosis and migration between treated and control groups were analyzed by a t-test. Differences in promoter methylation in the control experiment were evaluated using a Chi-square test. A P-value of $<0.05$ was considered to indicate a statistically significant difference.

\section{Results}

Gene screening. The methylation microarray from Illumina contains 27,578 highly informative $\mathrm{CpG}$ sites covering $>14,475$ human RefSeq genes. After SAM between high- and low-grade gliomas, with FDR of $<0.2,16,942$ probes were eliminated. Cox regression analysis left 66 probes. Gene expression profiling was used to screen 25,255 probes for differential expression between high- and low-grade gliomas by SAM. Intersection of the 66 probes set and the differentially expressed genes yielded 22 probes of interest. After univariate analysis validation using a t-test and Kaplan-Meier survival curves (by log-rank), two genes remained, PDE4C and CAMKK2. PDE4C was the gene we selected (Fig. 1).

Hypermethylation of $P D E 4 C$ is associated with grade progression in CGGA and The Cancer Genome Atlas (TCGA) data. We measured the methylation level of PDE4C in 119 glioma samples (63 low-grade gliomas, 33 anaplastic gliomas and 23 glioblastomas) via microarray. PDE4C promoter methylation level in the CGGA data had no significant difference on survival in grade II patients $(\mathrm{P}=0.4072)$ (Fig. 2A). Marginal $\mathrm{P}$-value in grade III patients is shown in Fig. $2 \mathrm{~B}(\mathrm{P}=0.0844)$. Significant at $\mathrm{P}<0.05$ in $\mathrm{GBM}$ and high grade patients in CGGA data is shown in Fig. 2C and D. When considering TCGA data, significant at $\mathrm{P}=0.036$ in $\mathrm{pGBM}$ patients is shown in Fig. 2E. PDE4C was also found markedly hypermethylated in high-grade gliomas $(\mathrm{P}=0.026)$ (Fig. $2 \mathrm{~F})$ Furthermore, we 

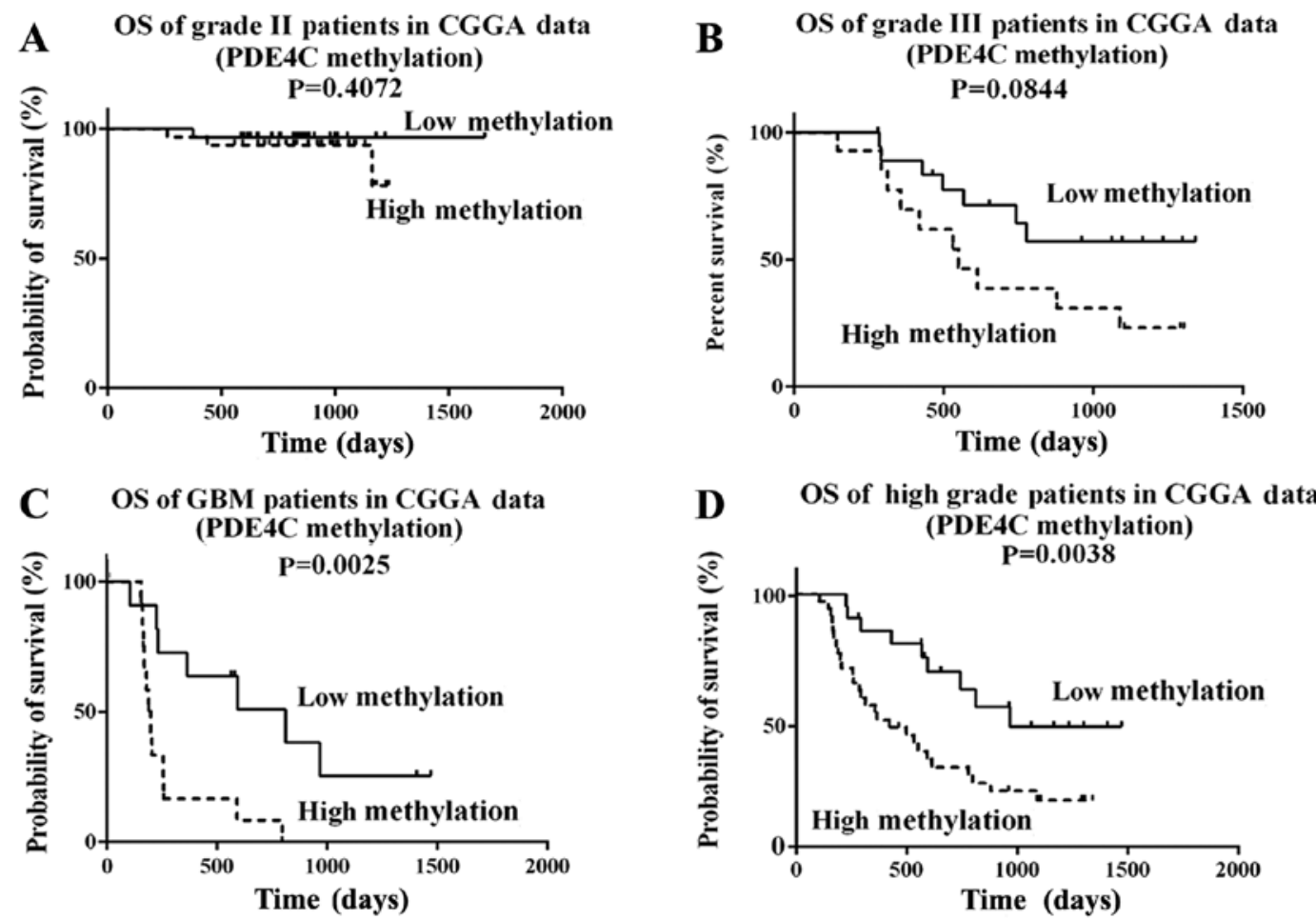

E OS of pGBM patients in TCGA data

$\mathbf{F}$

PDE4C methylation in CGGA data

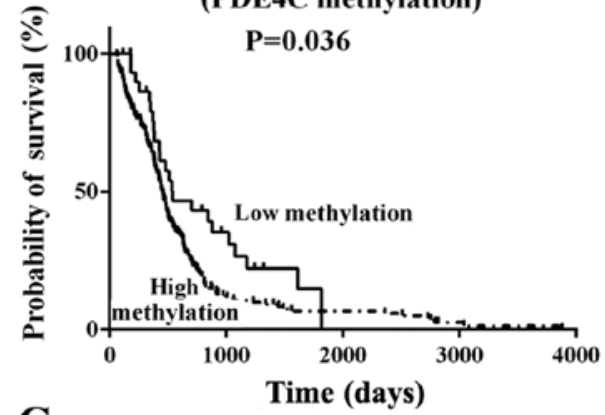

G
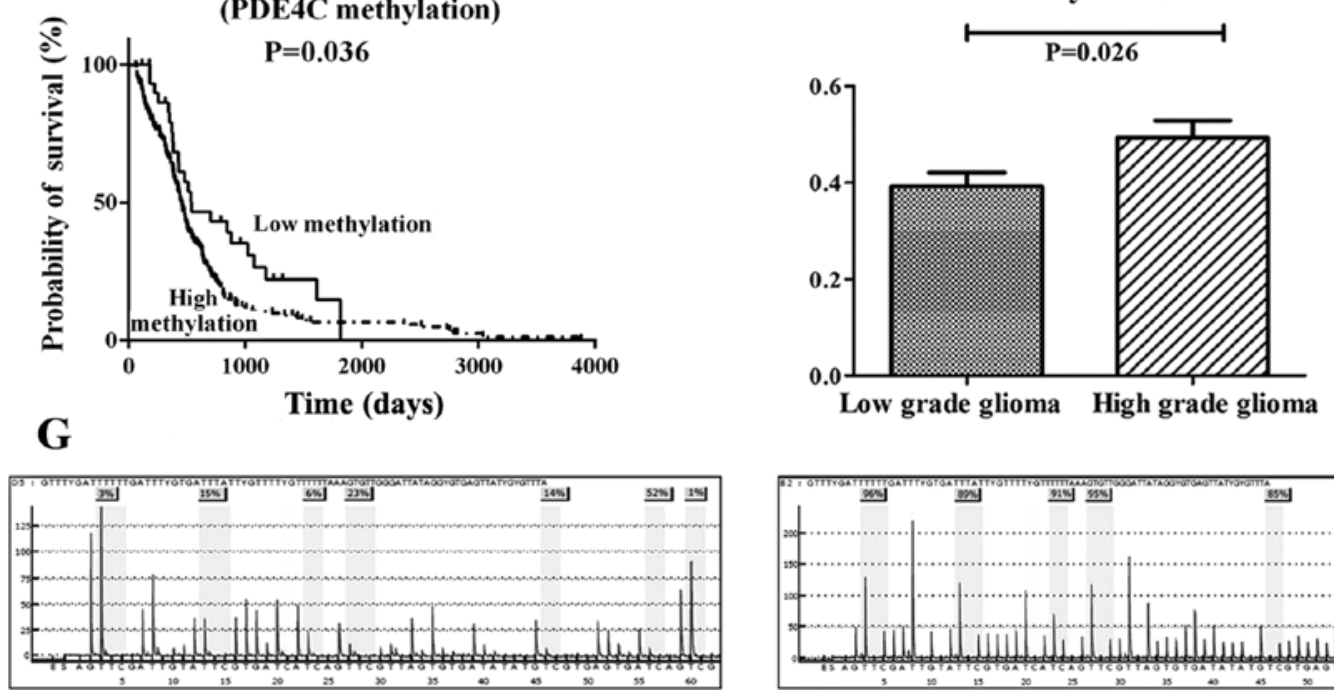

Hypomethylated PDE4C promoter

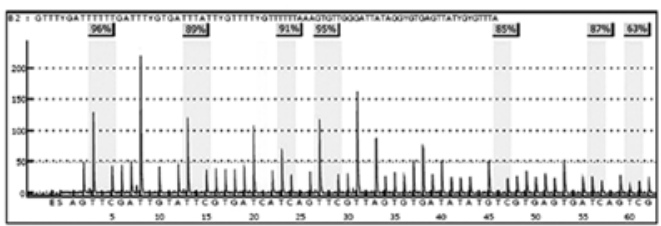

Hypermethylated PDE4C promoter

Figure 2. Promoter methylation decreases survival time of high-grade glioma patients. In high grade gliomas, patients with PDE4C hypermethylation levels had shorter OS than those hypomethylated in CGGA or TCGA database. (A) PDE4C promoter methylation level in the CGGA data had no significant difference on survival in grade II patients ( $\mathrm{P}=0.4072$ ). (B) Marginal $\mathrm{P}$-value in grade III patients $(\mathrm{P}=0.0844)$. (C and $\mathrm{D})$ Significant at $\mathrm{P}<0.01$ in $\mathrm{GBM}$ and high grade patients in CGGA data. (E) Significant at $\mathrm{P}<0.05$ in pGBM patients in TCGA data. (F) PDE4C promoter methylation levels were higher in high- than in low-grade glioma patients ( $\mathrm{P}=0.026)$. (G) Representative examples of PDE4C promoter methylation in low- and high-grade glioma patients. PDE4C, phosphodiesterase 4C; OS, overall survival; CGGA, Chinese Glioma Genome Atlas; TCGA, The Cancer Genome Atlas.

analyzed the independent prognostic value of PDE4C methylation. Patients with low PDE4C methylation levels had a longer median OS (not reached) compared with patients with high methylation levels (419 days) (Table I). Preoperative KPS score $(\mathrm{P}=0.004)$ and extent of resection $(\mathrm{P}=0.034)$ also correlated with OS. There were no significant associations between age, gender and OS. The multivariate Cox proportional hazards model, after adjusting for KPS score and extent of resection, identified PDE4C hypermethylation as an independent unfavorable prognostic factor $(\mathrm{P}=0.047)$.
Pyrosequencing analysis of PDE4C. Representative examples of PDE4C promoter methylation in glioma patients is shown in Fig. 2G. PDE4C promoter hypermethylation was detected in 29/37 high-grade cases (78.38\%), whereas just 12/18 low-grade samples $(66.67 \%)$ were hypermethylated. The methylation level of PDE4C promoter in the training and validation group was consistent $(\mathrm{P}=0.4905$, Chi-square test).

Expression of PDE4C is associated with grade progression in CGGA, TCGA and REMBRANDT data. Expression 
Table I. OS-related variables from 56 high-grade glioma methylation microarray univariate and multivariate analyses.

\begin{tabular}{|c|c|c|c|c|c|c|c|}
\hline \multirow[b]{2}{*}{ Variable } & \multicolumn{3}{|c|}{ Univariate analysis } & \multirow[b]{2}{*}{ P-value } & \multicolumn{3}{|c|}{ Multivariate analysis } \\
\hline & $\begin{array}{l}\text { No. of } \\
\text { patients }\end{array}$ & $\begin{array}{l}\text { Median OS } \\
\text { (days) }\end{array}$ & $\begin{array}{l}95 \% \text { CI } \\
\text { (days) }\end{array}$ & & $\begin{array}{l}\text { Relative } \\
\text { risk }\end{array}$ & $95 \% \mathrm{CI}$ & P-value \\
\hline \multicolumn{8}{|l|}{ Gender } \\
\hline Male & 23 & 591 & $248-745$ & & & & \\
\hline Female & 33 & 497 & $317-865$ & 0.528 & & & \\
\hline \multicolumn{8}{|c|}{ Age (years) } \\
\hline$\leq 50$ & 39 & 613 & $239-987$ & & & & \\
\hline$>50$ & 17 & 497 & $52-941$ & 0.084 & & & \\
\hline \multicolumn{8}{|l|}{ KPS } \\
\hline$<80$ & 19 & 290 & $135-445$ & & & & \\
\hline$\geq 80$ & 37 & 878 & NR & 0.004 & 0.452 & $0.210-0.973$ & 0.042 \\
\hline \multicolumn{8}{|c|}{ Extent of resection } \\
\hline Total & 17 & NR & NR & & & & \\
\hline Subtotal & 37 & 147 & $241-819$ & 0.034 & 2.424 & $0.981-5.993$ & 0.055 \\
\hline \multicolumn{8}{|c|}{ PDE4C-methylation } \\
\hline Low & 21 & NR & NR & & & & \\
\hline High & 35 & 419 & $160-678$ & 0.003 & 2.418 & $1.011-5.785$ & 0.047 \\
\hline
\end{tabular}

NR, not reached; CI, confidence interval; KPS, Karnofsky Performance Status; OS, overall survival; PDE4C, phosphodiesterase 4C.

profile analysis indicated PDE4C expression was decreased progressively in higher-grade gliomas compared with lowergrade gliomas. The CGGA patient cohort was the same for the gene expression dataset and methylation dataset. We found PDE4C expression level in the CGGA data had no significant difference on survival in grade II and III patients, $(\mathrm{P}=0.720$, $\mathrm{P}=0.31$ ) (Fig. 3A and $\mathrm{B}$ ). However, significant at $\mathrm{P}<0.05$ was shown in GBM patients in CGGA data $(\mathrm{P}<0.01)($ Fig. $3 \mathrm{C})$ and REMBRANDT ( $\mathrm{P}=0.0338$ ) (Fig. 3E). In CGGA and TCGA data, $P$-value was marginally significant in high grade patients in CGGA ( $\mathrm{P}=0.0797$ ) (Fig. 3D) or GBM patients in TCGA $(\mathrm{P}=0.0587)$ (Fig. 3F). PDE4C expression levels were lower in high- than in low-grade glioma patients $(\mathrm{P}<0.01)$ (Fig. 3G). GSVA analysis also showed that migration-related genes accumulated in patients with lower PDE4C expression, and apoptosis-related genes indicated the reverse (Fig. 3H).

Protein expression of PDE4C is inversely correlated with glioma-grade progression and shows prognostic value in high-grade glioma patients. We measured PDE4C protein levels through immunohistochemical staining in 87 paraffinembedded glioma samples and 5 normal brains from mainland Han Chinese glioma patients or traumatic brain injury patients. The 92 patients consisted of 5 brain trauma patients, 29 patients with astrocytoma (A, WHO grade II), 22 with anaplastic astrocytoma (AA, WHO grade III) and 36 with GBM (WHO grade IV). We analyzed the association between PDE4C protein expression and histological grade of gliomas. PDE4C protein was highly expressed in normal brains and lower grade gliomas compared with higher grade gliomas $(\mathrm{P}<0.001)$ (Fig. 4A and B). In normal brain, the percentage of PDE4C+ cells/tot nuclei was 55\%; in grade II glioma, the percentage was $49.33 \%$. In grade III glioma, the percentage was $6.25 \%$. In GBM, the percentage was only $1 \%$. In addition, survival analysis showed that patients with low PDE4C expression had notably poorer survival $(\mathrm{P}<0.001)$ than those with high expression in high-grade glioma patients (Fig. 4B).

Patients with high PDE4C expression levels had a longer median OS time (546 days) compared with those with low level expression (331 days) (Table II). Preoperative KPS score $(\mathrm{P}=0.025)$ and gender $(\mathrm{P}=0.028)$ also correlated with $\mathrm{OS}$. There were no significant associations with age or extent of resection and OS. The multivariate Cox proportional hazards model, after adjusting for gender and KPS score, identified PDE4C expression as an independent prognostic factor for OS $(\mathrm{P}=0.034)$.

PDE4C is associated with cell migration and $c A M P$-mediated signaling. Pearson correlation analysis of the CGGA mRNA expression data identified positively correlated genes that were then used for GO analysis. The top $10 \mathrm{GO}$ terms indicated PDE4C is associated with cell migration and cAMP-mediated signaling (Table III).

GSVA was also performed with PDE4C expression (Fig. 3H). Migration-related genes accumulated in patients with lower PDE4C expression. Based on previous studies of cAMP-P53 $(8,16)$, we also examined apoptosis-related genes and found they accumulated in patients with higher PDE4C expression.

Transcriptional silencing of PDE4C is associated with promoter hypermethylation in glioma cells. To determine whether PDE4C promoter hypermethylation in glioma is associated with PDE4C gene silencing, we measured PDE4C mRNA expression in three glioma cell lines. Semi-quantitative RT-PCR demonstrated that demethylation treatment by 

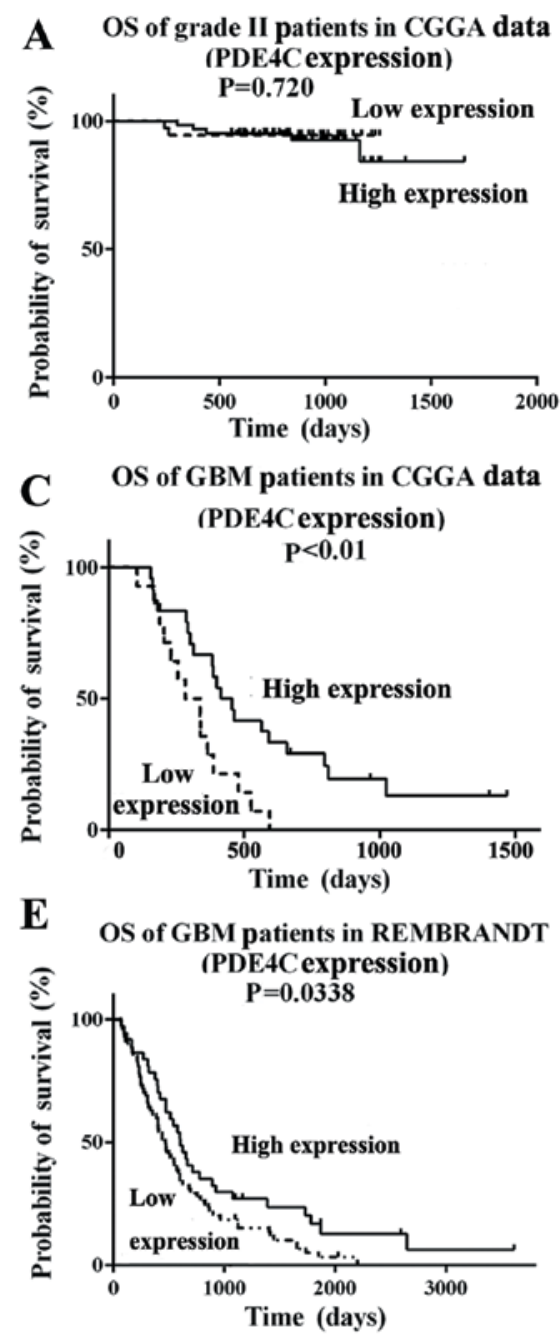

G

Differential expression level of PDE4C in

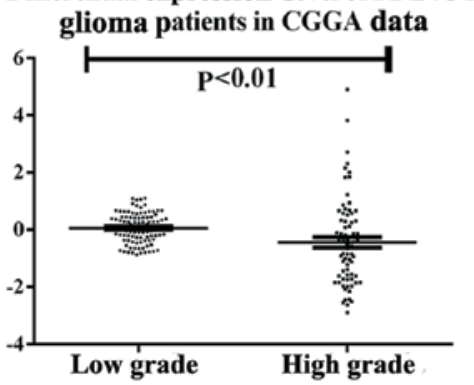

B OS of grade III patients in CGGA data (PDE4C expression)

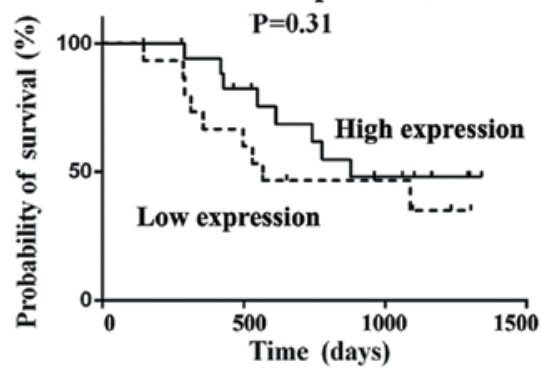

D OS of High grade glioma patients in CGGA data

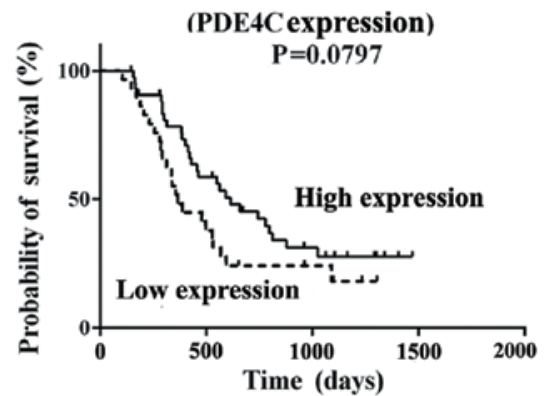

F OS of GBM patients in TCGA data

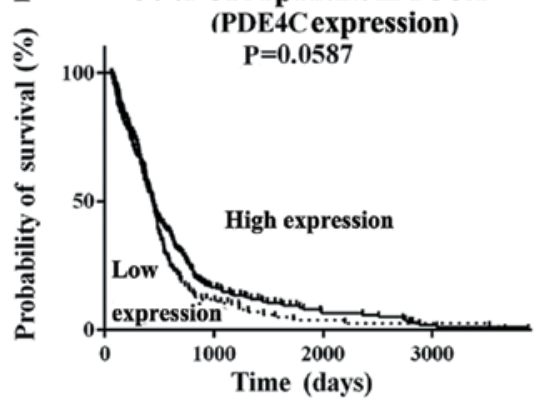

$\mathbf{H}$

Enrichment score Low High

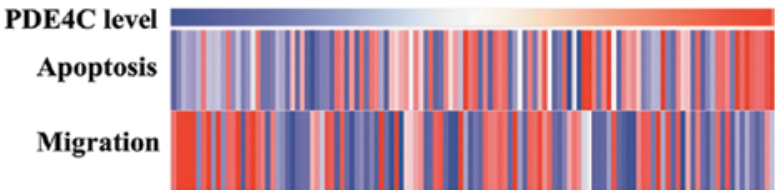

Figure 3. Reduced PDE4C expression correlates with glioma grade progression and prognosis. Expression profile analysis indicated PDE4C expression was decreased progressively in higher-grade gliomas compared with lower-grade gliomas. (A and B) PDE4C expression level in the CGGA data had no significant difference on survival in grade II and III patients $(\mathrm{P}=0.720, \mathrm{P}=0.31)$. (C and E) Significant at $\mathrm{P}<0.05$ in GBM patients in CGGA data $(\mathrm{P}<0.01)$ and REMBRANDT ( $\mathrm{P}=0.0338)$. ( $\mathrm{D}$ and $\mathrm{F}$ ) Marginal $\mathrm{P}$-value in high grade patients in CGGA ( $\mathrm{P}=0.0797)$ or in TCGA ( $\mathrm{P}=0.0587)$. (G) $\mathrm{PDE} 4 \mathrm{C}$ expression levels are lower in high- than in low-grade glioma patients $(\mathrm{P}<0.01)$. (H) GSVA analysis showed that migration-related genes accumulated in patients with lower PDE4C expression, and apoptosis-related genes indicated the reverse. PDE4C, phosphodiesterase 4C; CGGA, Chinese Glioma Genome Atlas; GSVA, gene set variation analysis.

5-Aza-2-dC restored PDE4C mRNA expression in glioma cells (U87, U251 and H4) (Fig. 5A), implicating DNA methylation in the regulation of PDE4C expression. Thus, these results indicate that transcriptional silencing of PDE4C in glioma cell lines may be mediated by DNA promoter hypermethylation.

PDE4C methylation influences WT p53 expression. To examine the mechanism by which PDE4C expression may regulate apoptosis through the WT p53 pathway, we knocked down restored PDE4C using siRNA successfully ( $\mathrm{P}=0.020$ vs. restored PDE4C) (Fig. 5B). We further investigated in protein level and observed that WT p53 downregulation was associated with PDE4C expression levels in the U87 cell line $(\mathrm{P}<0.01$, vs. control) (Fig. 5C). To confirm that P53 is increased due to the increase of PDE4C, we transfected pCMV6-Entry-PDE4C plasmid and control vectors into U87. Then, we confirmed that WT p53 upregulation is also dependent on PDE4C overexpression in the $\mathrm{U} 87$ cell line $(\mathrm{P}<0.05)$ (Fig. 5D). 

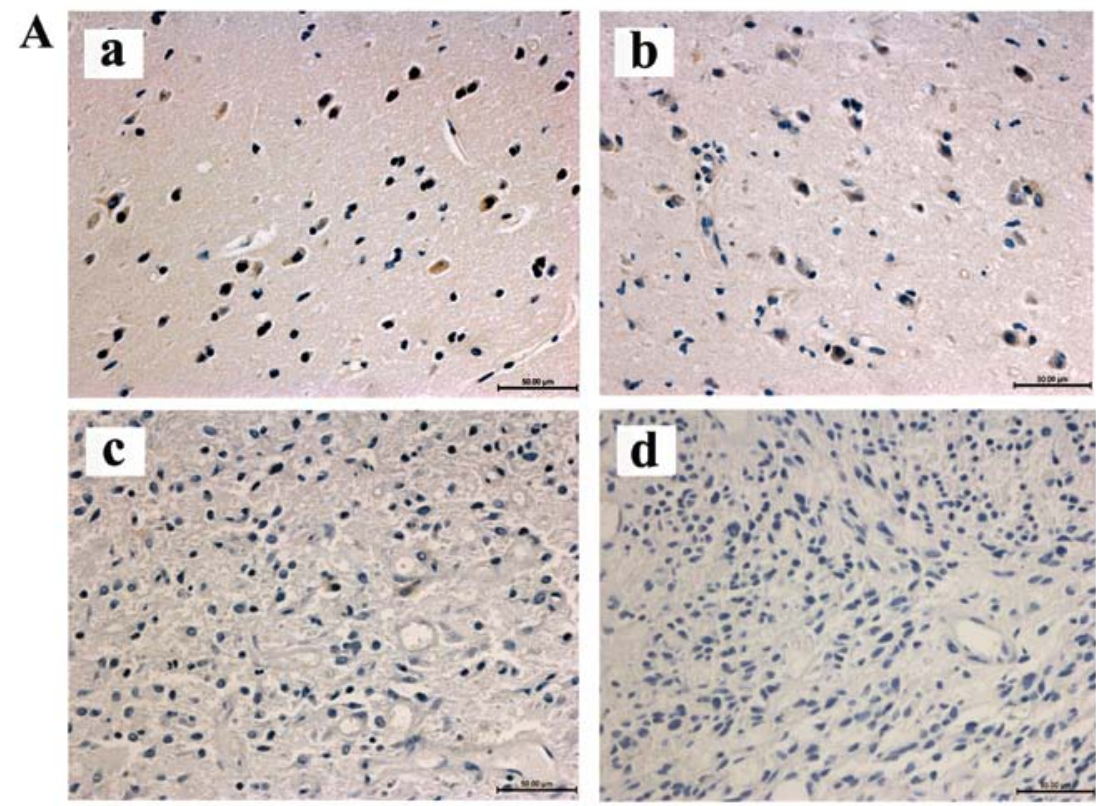

B a

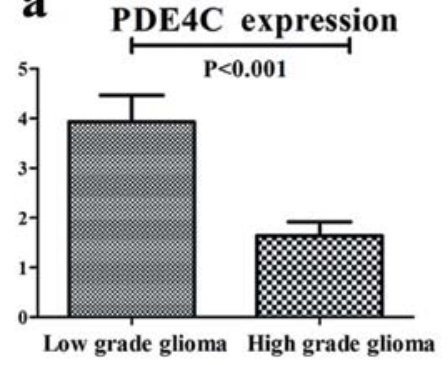

b

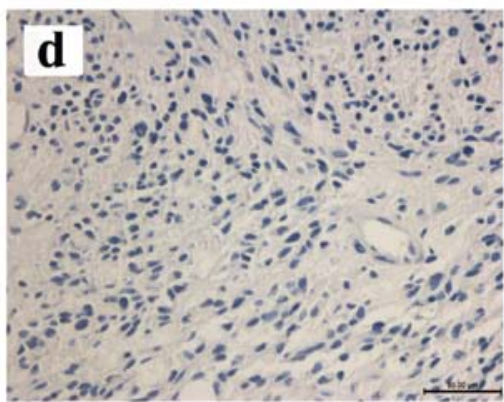

b High grade glioma overall survival

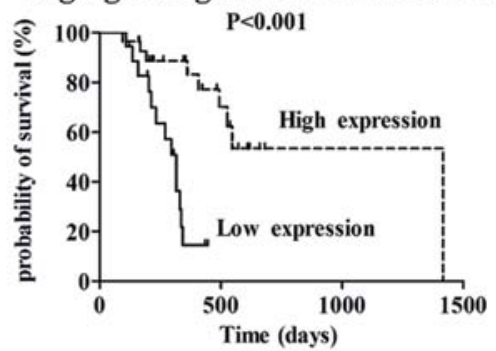

Figure 4. PDE4C expression is decreased progressively in high-grade gliomas compared with low-grade gliomas. (A) Immunohistochemical staining indicates PDE4C expression correlates with glioma grade progression and prognosis. (A-a) Normal brain tissue; (A-b) astrocytoma (WHO grade II); (A-c) anaplastic astrocytoma (WHO grade III) and (A-d) glioblastoma (WHO grade IV). (B) Analysis using the IHC data. (B-a) PDE4C expression is lower in high- than in low-grade glioma $(\mathrm{P}<0.001)$; (B-b) high-grade glioma patients with high PDE4C expression had significantly longer OS than those with low expression $(\mathrm{P}<0.001)$. PDE4C, phosphodiesterase 4C.

$\mathbf{A}$

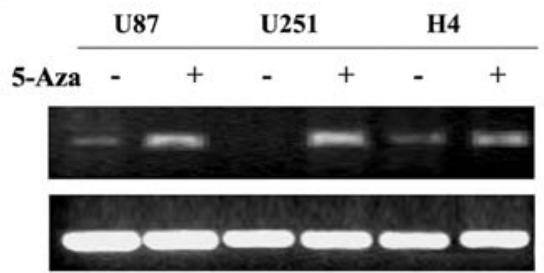

C

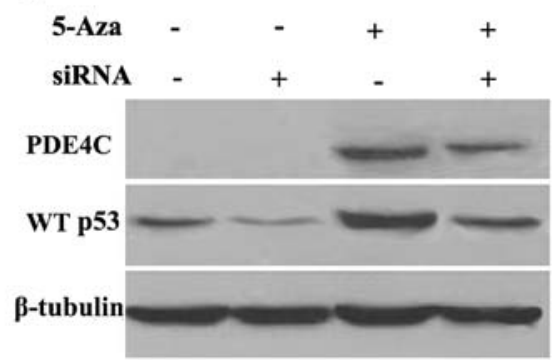

B

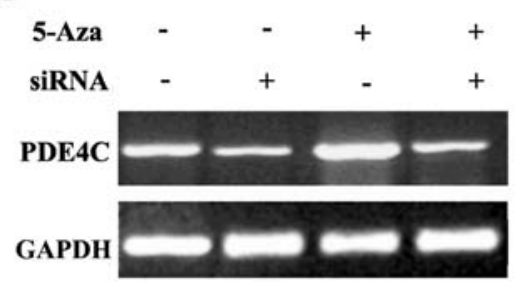

D

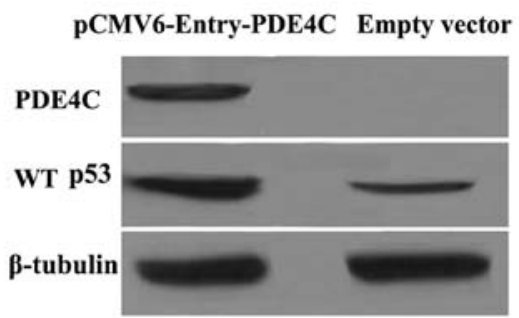

Figure 5. PDE4C expression correlates with promoter hypermethylation and influences WT p53 expression. (A) PDE4C expression is restored by 5-Aza as indicated by RNA level. (B) Restored PDE4C expression is knocked down by siRNA ( $\mathrm{P}<0.05$ vs. control). (C and D) Restored or overexpressed PDE4C influences WT p53 expression at the protein level (western blotting identified, $\mathrm{P}<0.05$ vs. control). PDE4C, phosphodiesterase 4C; siRNA, small interfering RNA. 
Table II. OS-related variables from 46 (45 survivors) high-grade glioma samples validated through univariate and multivariate analyses.

\begin{tabular}{|c|c|c|c|c|c|c|c|}
\hline \multirow[b]{2}{*}{ Variable } & \multicolumn{3}{|c|}{ Univariate analysis } & \multirow[b]{2}{*}{ P-value } & \multicolumn{3}{|c|}{ Multivariate analysis } \\
\hline & $\begin{array}{l}\text { No. of } \\
\text { patients }\end{array}$ & $\begin{array}{l}\text { Median OS } \\
\quad \text { (days) }\end{array}$ & $\begin{array}{c}95 \% \text { CI } \\
\text { (days) }\end{array}$ & & $\begin{array}{l}\text { Relative } \\
\text { risk }\end{array}$ & $95 \% \mathrm{CI}$ & P-value \\
\hline \multicolumn{8}{|l|}{ Gender } \\
\hline Male & 31 & 572 & $380-764$ & & & & \\
\hline Female & 14 & 345 & $238-451$ & 0.028 & 0.544 & $0.224-1.323$ & 0.179 \\
\hline \multicolumn{8}{|c|}{ Age (years) } \\
\hline$\leq 50$ & 22 & 572 & $387-757$ & & & & \\
\hline$>50$ & 24 & 354 & $320-388$ & 0.273 & & & \\
\hline \multicolumn{8}{|c|}{ Extent of resection } \\
\hline Total & 16 & 345 & $207-483$ & & & & \\
\hline Untotal & 29 & 493 & $360-626$ & 0.196 & & & \\
\hline \multicolumn{8}{|l|}{ KPS } \\
\hline$<80$ & 28 & 362 & $189-535$ & & & & \\
\hline$\geq 80$ & 17 & 572 & $389-755$ & 0.025 & 0.510 & $0.181-1.432$ & 0.201 \\
\hline \multicolumn{8}{|c|}{ PDE4C-expression } \\
\hline Low & 18 & 331 & 278-384 & & & & \\
\hline High & 28 & 546 & $433-659$ & 0.004 & 0.345 & $0.129-0.923$ & 0.034 \\
\hline
\end{tabular}

CI, confidence interval; KPS, Karnofsky Performance Status; OS, overall survival; PDE4C, phosphodiesterase 4C.

Table III. Gene sets enriched in high-grade glioma samples with PDE4C expression.

\begin{tabular}{lccr}
\hline Name & Count & Fold enrichment & P-value \\
\hline GO:0005576 - extracellular region & 804 & 1.390481 & $1.63 \mathrm{E}-32$ \\
GO:0031226 - intrinsic to plasma membrane & 517 & 1.479175 & $2.26 \mathrm{E}-27$ \\
GO:0005887 - integral to plasma membrane & 506 & 1.480605 & $6.92 \mathrm{E}-27$ \\
GO:0016021 - integral to membrane & 1789 & 1.174047 & $2.78 \mathrm{E}-26$ \\
GO:0005886 - plasma membrane & 1331 & 1.225 & $1.16 \mathrm{E}-25$ \\
GO:0044421 - extracellular region part & 374 & 1.354271 & $1.65 \mathrm{E}-12$ \\
GO:0005615 - extracellular space & 275 & 1.395556 & $5.71 \mathrm{E}-11$ \\
hsa04630 - Jak-STAT signaling pathway & 76 & 1.774584 & $1.88 \mathrm{E}-08$ \\
GO:0019933 - cAMP-mediated signaling & 50 & 1.89816 & $6.99 \mathrm{E}-07$ \\
GO:0005230 - extracellular ligand-gated ion channel activity & 41 & 2.038913 & $7.28 \mathrm{E}-07$ \\
GO:0016338 - calcium-independent cell-cell adhesion & 17 & 2.786845 & $1.46 \mathrm{E}-05$ \\
\hline
\end{tabular}

PDE4C, phosphodiesterase 4C.

Ectopic expression of PDE4C induces U87 cell apoptosis and inhibits migration. In the present study, we observed a difference in U87 apoptosis $48 \mathrm{~h}$ following transient transfection with PDE4C and control vectors using Annexin V-PI flow cytometry, and compared with untreated U87 cells $(\mathrm{P}<0.05)$ (Fig. 6). Region Q2 indicates early-phase apoptotic cells, whereas region Q4 indicates late-phase apoptotic cells. Cells were further examined using the Transwell migration assay, which showed that PDE4C inhibited U87 invasion $(\mathrm{P}<0.05)$ (Fig. 7). All experiments were performed in triplicate.

\section{Discussion}

Survival-associated genes in gliomas have been reported in various studies (21-24). Moreover, methylation microarray provides a more general, prognostic, genome-wide methylation profiling, which may have a role in individualized diagnosis and treatment. In addition, DNA methylation is stable and can be detected using a number of high-throughput and sensitive techniques with little patient material (25).

In the present study, we identified an epigenetically silenced gene, PDE4C, which may serve as a biomarker for 


\section{Control}

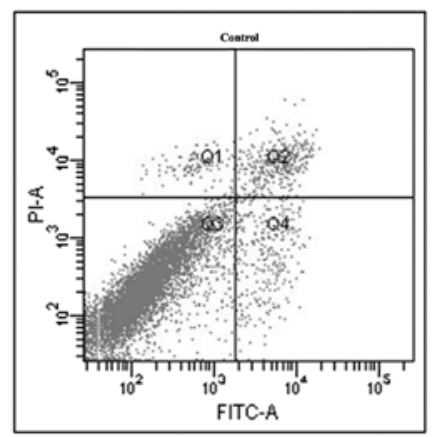

Empty vector

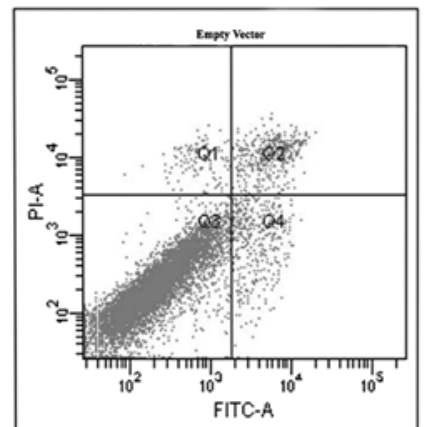

pCMV6-Entry-PDE4C

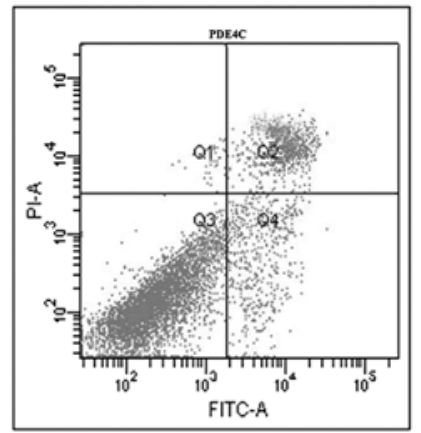

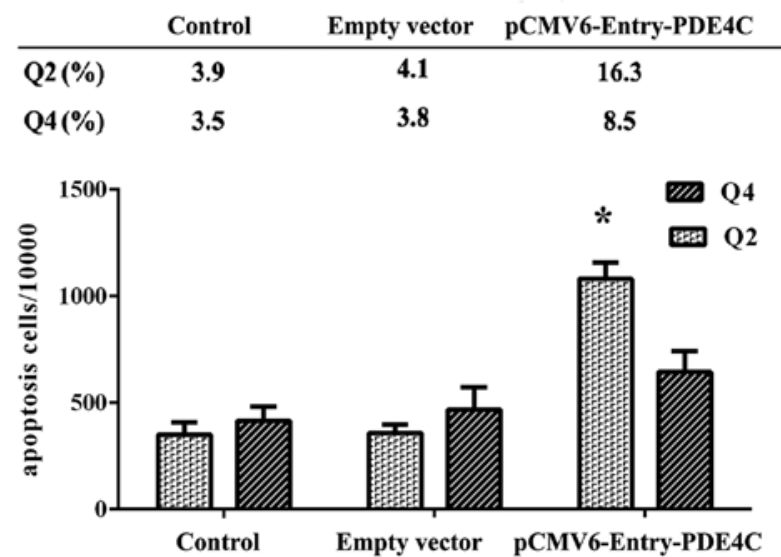

Figure 6. PDE4C induces apoptosis of U87 cell line. Cell apoptosis was detected by the Annexin V-PI flow cytometry assay. U87 cells transfected with PDE4C induced apoptosis compared with untreated groups or groups treated with an empty vector. Region Q4 indicates early-phase apoptotic cells, whereas Q2 shows late-phase apoptotic cells ( ${ }^{*} \mathrm{P}=0.0316$ vs. control). PDE4C, phosphodiesterase 4C.

\section{Control}

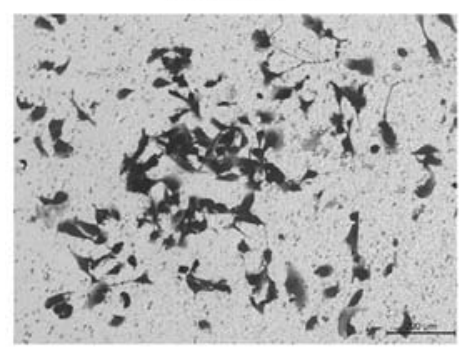

Empty vector

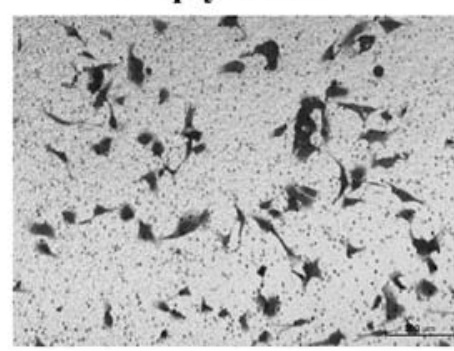

pCMV6-Entry-PDE4C

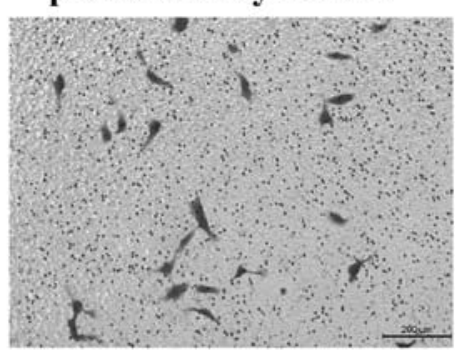

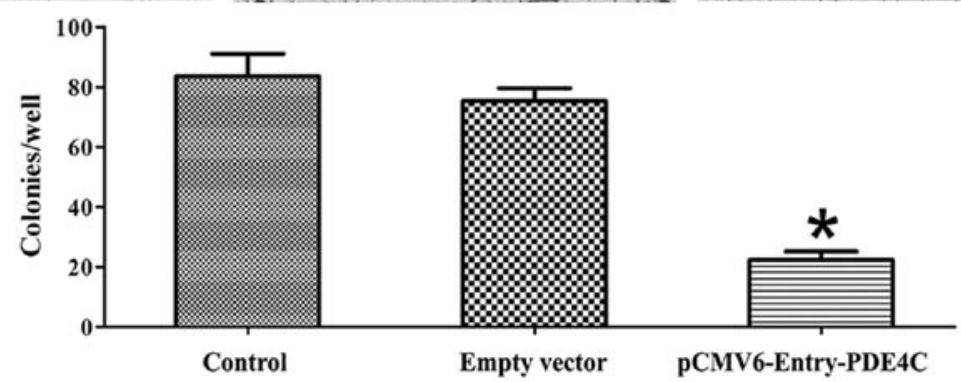

Figure 7. PDE4C inhibits invasion of U87 cell line. Invasion of U87 cell lines was assessed by Transwell assay. The number of invading cells was significantly less in the PDE4C group compared with the empty vector or untreated group. Number of invading cells shown as a histogram ("P<0.05 vs. control). PDE4C, phosphodiesterase $4 \mathrm{C}$.

glioma progression and prognosis. Primarily, we carried out genome-wide DNA profiling and found that PDE4C promoter methylation levels are associated with glioma grade progression. When correlated with data from gene expression profiling, we identified PDE4C as differentially expressed in low- and high-grade gliomas. We also found that promoter methylation and PDE4C expression levels were consistently associated with glioma grade progression. To confirm this finding, we showed that PDE4C transcription in glioma was associated with promoter demethylation. We corroborated this 
finding by pyrosequencing a large cohort of samples (total of $\mathrm{n}=55$ ), and showed that glioma PDE4C expression is negatively associated with WHO grade classification. Protein expression was examined in 87 glioma patient samples and five normal brain tissues by immunohistochemical methods, which demonstrated a significant decrease in PDE4C expression in low- and high-grade gliomas. The results showed that both PDE4C promoter methylation and PDE4C expression were independent prognostic factors in high-grade gliomas, and revealed a correlation between PDE4C promoter methylation, PDE4C expression and clinical outcome in glioma patients.

Previous studies have shown that in malignant cells, cAMP levels influence p53 function, potentially effecting WT p53 both as a tumor suppressor during cancer initiation and maintenance, and as an effector of the apoptotic response to DNA-damaging agents during anticancer treatment (8). PDE4s may be involved in this progression. Our western blot analysis revealed that WT p53 expression is associated with PDE4C expression in U87 cell lines. To show that WT p53 expression is regulated by PDE4C, we used PDE4C knockdown using siRNA to restore WT p53 downregulation and overexpression of PDE4C also influenced WT p53 expression. Our GO and GSVA analysis, combined with PDE4C functional assays in U87 cell lines, strongly showed that PDE4C induces apoptosis and inhibits glioma migration. To the best of our knowledge, this is the first report on the methylation status, expression and function of PDE4C in glioma.

In summary, PDE4C promoter hypermethylation is associated with low PDE4C expression. Patients harboring hypermethylation and low-level PDE4C expression have a poor prognosis in high-grade glioma. On the basis of these observations and subset analysis, it appears that PDE4C promoter methylation and PDE4C expression can both serve as prognostic biomarkers for glioma patients. PDE4C-induced apoptosis in glioma appears to involve the cAMP-P53 pathway, although further research is required to fully elucidate the role of PDE4C in cell migration.

\section{Acknowledgements}

The authors thank Dr Susan Furness for her critical reading of the manuscript, Dr Wei Zhang and Dr Zheng Wang for their help on microarray data analysis of this project. This study was supported by grants from the National High Technology Research and Development Program (no. 2012AA02A508), the International Science and Technology Cooperation Program (no. 2012DFA30470), the National 973 Program (no. 2011CB707804), and the National Natural Science Foundation of China nos. 91229121,81201993 and 81071626.

\section{References}

1. Krex D, Klink B, Hartmann C, et al: Long-term survival with glioblastoma multiforme. Brain 130: 2596-2606, 2007.

2. Zhang W, Yan W, You G, et al: Genome-wide DNA methylation profiling identifies ALDH1A3 promoter methylation as a prognostic predictor in G-CIMP- primary glioblastoma. Cancer Lett 328: 120-125, 2013.

3. Maegawa S, Yoshioka H, Itaba N, et al: Epigenetic silencing of PEG3 gene expression in human glioma cell lines. Mol Carcinog 31: 1-9, 2001.
4. van den Boom J, Wolter M, Kuick R, et al: Characterization of gene expression profiles associated with glioma progression using oligonucleotide-based microarray analysis and real-time reverse transcription-polymerase chain reaction. Am J Pathol 163: 1033-1043, 2003.

5. Hu Y, Pioli PD, Siegel E, et al: EFEMP1 suppresses malignant glioma growth and exerts its action within the tumor extracellular compartment. Mol Cancer 10: 123, 2011.

6. He SM, Zhao ZW, Wang Y, et al: Reduced expression of SMAD4 in gliomas correlates with progression and survival of patients. J Exp Clin Cancer Res 30: 70, 2011.

7. Grønbaek K, Hother $C$ and Jones PA: Epigenetic changes in cancer. APMIS 115: 1039-1059, 2007.

8. Naderi EH, Findley HW, Ruud E, Blomhoff HK and Naderi S: Activation of cAMP signaling inhibits DNA damage-induced apoptosis in BCP-ALL cells through abrogation of p53 accumulation. Blood 114: 608-618, 2009.

9. Foltz G, Ryu GY, Yoon JG, et al: Genome-wide analysis of epigenetic silencing identifies $B E X 1$ and $B E X 2$ as candidate tumor suppressor genes in malignant glioma. Cancer Res 66: 6665-6674, 2006.

10. Kim YJ, Yoon HY, Kim JS, et al: HOXA9, ISL1 and ALDH1A3 methylation patterns as prognostic markers for nonmuscle invasive bladder cancer: array-based DNA methylation and expression profiling. Int J Cancer 133: 1135-1142, 2013.

11. Berdasco M, Ropero S, Setien F, et al: Epigenetic inactivation of the Sotos overgrowth syndrome gene histone methyltransferase NSD1 in human neuroblastoma and glioma. Proc Natl Acad Sci USA 106: 21830-21835, 2009.

12. Jin SL, Ding SL and Lin SC: Phosphodiesterase 4 and its inhibitors in inflammatory diseases. Chang Gung Med J 35: 197-210, 2012.

13. Beavo JA and Reifsnyder DH: Primary sequence of cyclic nucleotide phosphodiesterase isozymes and the design of selective inhibitors. Trends Pharmacol Sci 11: 150-155, 1990.

14. Conti M, Jin SL, Monaco L, Repaske DR and Swinnen JV: Hormonal regulation of cyclic nucleotide phosphodiesterases. Endocr Rev 12: 218-234, 1991.

15. Pérez-Torres S1, Miró X, Palacios JM, Cortés R, Puigdoménech P and Mengod G: Phosphodiesterase type 4 isozymes expression in human brain examined by in situ hybridization histochemistry and $\left[{ }^{3} \mathrm{H}\right]$ rolipram binding autoradiography. Comparison with monkey and rat brain. J Chem Neuroanat 20: 349-374, 2000.

16. Naderi EH, Jochemsen AG, Blomhoff HK and Naderi S: Activation of cAMP signaling interferes with stress-induced p53 accumulation in ALL-derived cells by promoting the interaction between p53 and HDM2. Neoplasia 13: 653-663, 2011.

17. Li S, Yan C, Huang L, Qiu X, Wang Z and Jiang T: Molecular prognostic factors of anaplastic oligodendroglial tumors and its relationship: a single institutional review of 77 patients from China. Neuro Oncol 14: 109-116, 2012.

18. Hill VK, Ricketts C, Bieche I, et al: Genome-wide DNA methylation profiling of $\mathrm{CpG}$ islands in breast cancer identifies novel genes associated with tumorigenicity. Cancer Res 71: 2988-2999, 2011.

19. Cadieux B, Ching TT, VandenBerg SR and Costello JF: Genome-wide hypomethylation in human glioblastomas associated with specific copy number alteration, methylenetetrahydrofolate reductase allele status, and increased proliferation. Cancer Res 66: 8469-8476, 2006.

20. Owens RJ, Lumb S, Rees-Milton K, et al: Molecular cloning and expression of a human phosphodiesterase 4C. Cell Signal 9: 575-585, 1997.

21. Czernicki T, Zegarska J, Paczek L, et al: Gene expression profile as a prognostic factor in high-grade gliomas. Int $\mathbf{J}$ Oncol 30: 55-64, 2007.

22. Freije WA, Castro-Vargas FE, Fang Z, et al: Gene expression profiling of gliomas strongly predicts survival. Cancer Res 64: 6503-6510, 2004.

23. Takahashi H and Kouno J: Gene expression profiling of malignant gliomas by cDNA microarrays. Nihon Rinsho 63 (Suppl 9): 515-519, 2005 (In Japanese).

24. Yoshino A, Ogino A, Yachi K, et al: Gene expression profiling predicts response to temozolomide in malignant gliomas. Int $\mathbf{J}$ Oncol 36: 1367-1377, 2010.

25. Schatz P, Distler J, Berlin K and Schuster M: Novel method for high throughput DNA methylation marker evaluation using PNA-probe library hybridization and MALDI-TOF detection. Nucleic Acids Res 34: e59, 2006. 